\title{
Bekenntnisse eines Unbequemen
}

„Bürger und Irre“, „Irren ist menschlich“ - mit solchen Werken hat sich Klaus Dörner als Fundamentalkritiker der Psychiatrie einen Namen gemacht. Als Leiter der Psychiatrischen Klinik in Gütersloh hat er in den 1990er-Jahren die Auflösung der Abteilung für chronisch Kranke betrieben und so bewiesen, dass niemand auf Dauer in einer Anstalt leben muss. Auch mit 82 Jahren ist er seiner Methode treu geblieben: der kritischen Selbstwahrnehmung. Der PPH gewährte er in seiner Wohnung in HamburgEppendorf sehr persönliche Einblicke.

Chris Bleher

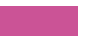

Der Kampf um die Humanisierung der Psychiatrie verlangt Rückgrat. Für Besucher im Hause Dörner gilt das wörtlich: Während der 82 Jahre alte Professor im Arbeitszimmer der großzügigen Eppendorfer Altbauwohnung erklärt, wie er seit Jahrzehnten dickste Bretter bohrt, versinkt man in der Tiefe des braunen Breitcord-Polstersessels, beugt sich gebannt nach vorn und spürt irgendwann dieses Ziehen im Kreuz. Und in der Lunge. Nach mehrstündigem Kolloquium ist die warme Luft gesättigt vom Rauch der Pfeife. Wohl dem, der selbst qualmt.

Aber wer hat gesagt, dass die Begegnung mit diesem blitzwachen Gesinnungstäter bequem und angenehm geraten müsse? So viel wusste man schon vorher: Klaus Dörner, Doktor der Medizin und der Philosophie, Studium der Soziologie und der Geschichte, Verfasser des Standardwerks „Bürger und Irre“, Mitautor des Psychiatrie-Bestsellers „Irren ist menschlich“, Leiter der Westfälischen Klinik für Psychiatrie in Gütersloh von 1981 bis zur Pensionierung 1996, unerbittlicher Gegner der Verwahrung chronisch Kranker in Anstalten, Impulsgeber für zahlreiche Bürgerund Nachbarschaftsinitiativen der ambulanten, kommunalen Versorgung pflegebedürftiger Menschen, Vortragsreisender mit einem Pensum von rund 200 Auftritten pro Jahr - dieser Mann des Geistes wie der Aktion verlangt seinen Mitmenschen das Äußerste ab im Namen der Menschlichkeit: Die Konfrontation mit sich selbst.

Und wie könnte man ihm das verübeln, wo er doch auch sich selbst nicht schont. Schon physisch nicht. Zu seinen Vorträgen

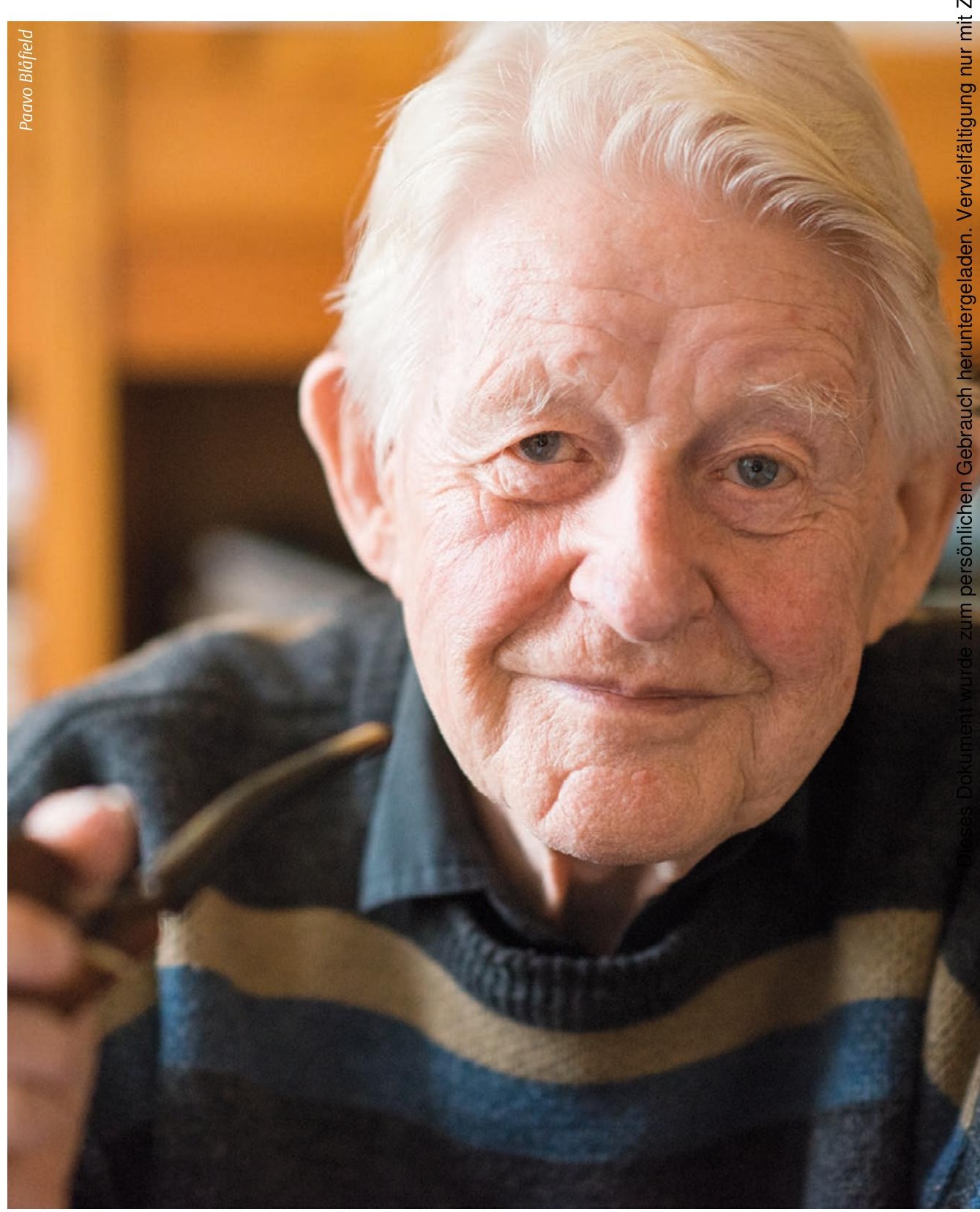



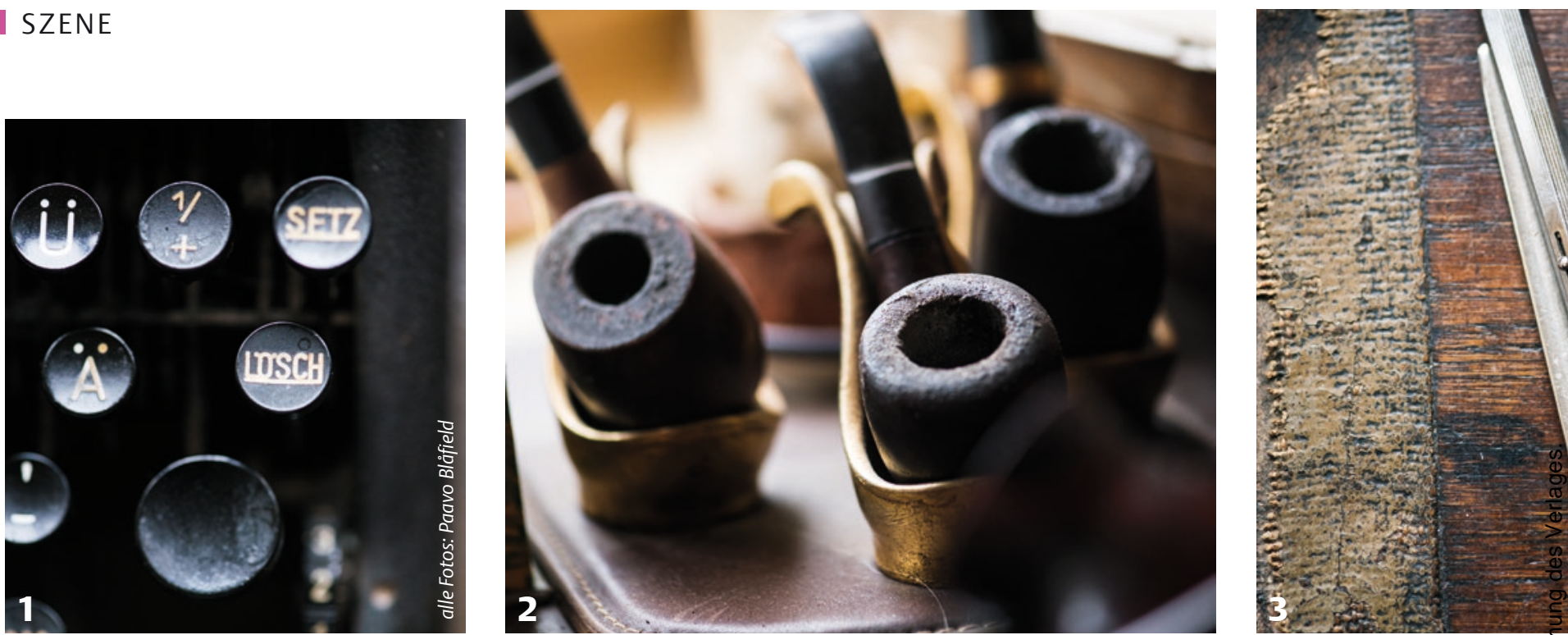

reist er stets mit der Bahn, zweiter Klasse; statt sich ins Taxi zu setzen, geht er zu Fuß; zwischen zwei Auftritten fährt er lieber heim zu seiner Frau, als im schnell erreichbaren Hotel zu übernachten, er hat ja die Bahncard 100; seinen Kaffee trinkt er schwarz, und vor einem Vortrag isst er nichts. Würde nur belasten.

\section{Dörnern - man ver- wendete dieses Verb} augenzwinkernd, als Warnung vor Übermotivation.

Zum 80. haben die drei Verlage, in denen er publiziert, diesem eigenwilligen Mann ein Büchlein mit 80 Dankesbriefen von Menschen geschenkt, in deren Institutionen er zu Gast war. „Herr Dörner kommt mit dem Zug“, heißt es. Darin sind bewegende Dankesworte zu lesen über diesen „wichtigen Wegweiser“, den „Wortzauberer“, den „Guru der Sozialpsychiatrie“, den „Visionär“. Einer schrieb: „Anfang der 1990er gab es das Verb ,dörnern' auf psychiatrischen Stationen. Man verwendete es augenzwinkernd als Warnung vor Übermotivation.“

Nicht nur mit sich, auch mit dem Denkmal seiner selbst geht Dörner schonungslos um. Den Besuch der PPH nutzt der fünffache Vater und zehnfache Großvater, heftig daran zu rütteln, statt es zu polieren. Die Stunden in der Wohnung in Hamburg-Eppendorf geraten zu einer heiteren Demythologisierung seiner selbst und seines fundamental-kritischen Werks. „Man könnte es aber auch genau andersherum sehen“ - wenn er das sagt, geht es ihm freilich nicht ums pure Relativieren. Er will

Horizonte öffnen, Denken als Probehandlung erproben. Und nach wie vor die Welt verbessern. Zumindest den Teil, auf den er Einfluss hat - die Pflege und Betreuung alter Leute und psychisch Kranker.

\section{Langzeitpatienten als Geiseln der Heime und ihrer Träger.}

„Irren ist menschlich“, der Titel erweist sich während des Besuchs als Dörners Grundhaltung. Er rechnet mit der eigenen Fehlbarkeit. Mehr als einmal wird Dörner an diesem Tag betonen, was gut, aber nicht gut genug gelaufen ist, was Glück war oder $\mathrm{Zu}$ fall. Kein Widerspruch, dass sich das 1978 erschienene Werk 400000 mal verkauft hat und er gerade an der 24. Auflage sitzt. Der Erfolg hat eben mit der Methode zu tun, die ihm zur zweiten Natur geworden ist: Das selbstkritische Erkunden der Landschaft, in der man therapeutisch tätig wird. Wie könnte das je an ein Ende kommen?

Generationen von psychiatrisch Tätigen und Betroffenen haben darin leicht verständliche, herausfordernde Sätze gelesen. Im Vorwort zur aktuellen Auflage geht es ums Ganze: „Vor allem unser Verstehensbegriff, unser Bemühen, den psychisch Kranken als Anderen zu verstehen, uns in ihn einzufühlen, achtet nicht immer radikal genug die Andersartigkeit, Fremdheit und damit die Würde des Anderen.“

Es sei zu fragen, ob ,unser bisheriger Ansatz einer nochmaligen strengen kritischen Prüfung standhält". Und statt es bei abstrakten Worten zu belassen, wird tiefer hineingebohrt in die Wunde im eigenen Fleisch:
„Es spricht auch nicht gerade für uns, dass wir bisher für die psychisch Kranken, gerade wo sie am schwächsten und ausgegrenztesten sind, die geringste Aufmerksamkeit übrig hatten.“ Die Rede sei von den „psychisch kranken Straftätern im Maßregelvollzug“ und von den „Langzeitpatienten“. Die habe man bis in die heutige Zeit hinein von „Geiseln der Landeskrankenhäuser“ zu „Geiseln der Heime und ihrer Träger“ gemacht.

Die Befreiung dieser „Geiseln“ aus Gründen der Menschlichkeit, das war Dörners erklärtes Ziel, als er und Suse, seine zweite Frau, eine gelernte Krankenschwester, von Hamburg nach Gütersloh gingen. Und tatsächlich war nach gut 15 Jahren auch der letzte der 435 chronisch psychisch Kranken entlassen. In „Ende der Veranstaltung - Anfänge der ChronischKranken-Psychiatrie“ haben Dörner und einige Mitstreiter beschrieben, wie das gelang, und wie es dann mit den Patienten weiterging. Es lieferte den Beweis: Chronisch psychisch Kranke brauchen kein stationäres Heilsystem.

\section{Wir haben über Generationen hinweg grob fahrlässig Freiheits- beraubung betrieben.}

Doch jenes Buch, auf das er stolz ist, weil es Resultat der „eigenen, langjährigen handfesten Arbeit in der Psychiatrie“ ist, kam am wenigsten an. Dörner redet nichts schön. Er sagt: „Mangels Interesse ist es nicht gekauft worden." Und korrigiert sich gleich selbst: „Es hat zu sehr gegen die gesamte Psychiatrie-Tradition verstoßen.“ 

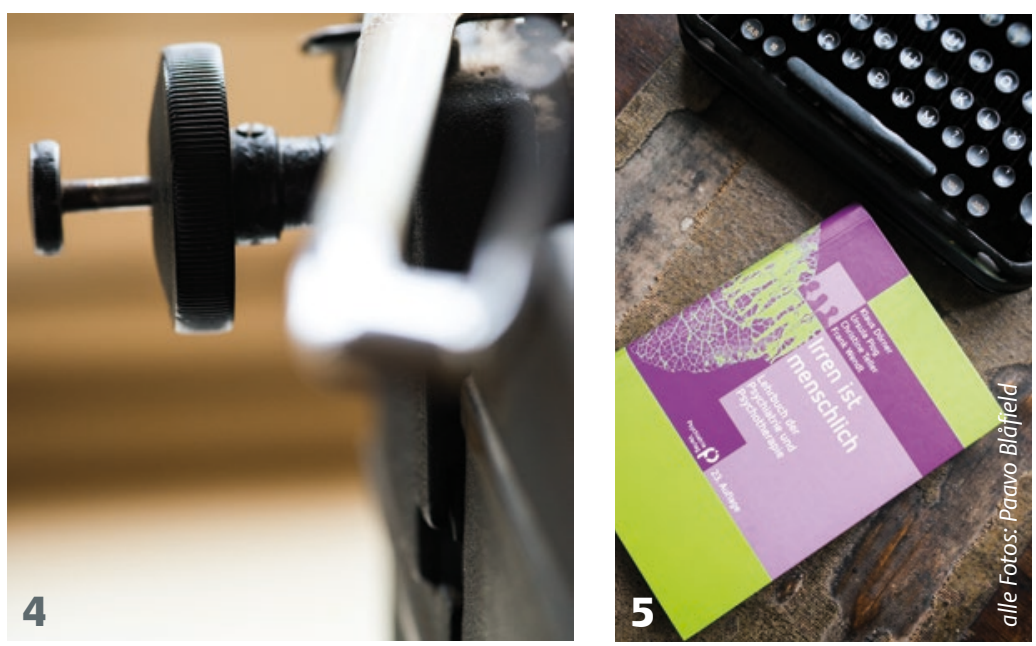

Abb. 1 Von wegen „delete“: Ausgemustert wird das funktionstüchtige Schreibgerät ...

Abb. 2 ... ebenso wenig wie die eingerauchten Exemplare desselben Typs Pfeife ...

Abb. 3 ... oder die gut durchgearbeitete Schreibunterlage.

Abb. 4 Was aus dieser analogen Welt heraustransportiert wird ...

Abb. 5 ... ist so brisant und aktuell wie eh und je.

Was der gebürtige Duisburger, Sohn eines praktischen Arztes und Geburtshelfers, in Gütersloh ins Werk gesetzt hat, das war die radikale Fortsetzung der Psychiatrie-Enquête von 1975. Die sah Dörner als „zu sehr vom Akut-Kranken her“ gedacht. 1988 hat eine Expertenkommission der Bundesregierung zur Psychiatriereform eine Empfehlung zugunsten einer Gemeindepsychiatrie abgegeben, die habe von den chronisch Kranken auszugehen. Nur: Passiert ist es nicht.

Dann kommt einer dieser donnernden Dörner-Sätze, in ruhigem Ton vorgebracht: „Wir alle haben über Generationen hinweg grob fahrlässig Freiheitsberaubung betrieben. Und uns dafür auch noch Bundesverdienstkreuze und andere Orden anstecken lassen." Hunderttausende wurden Dörner zufolge auf diese Weise lediglich in Heime „umhospitalisiert“. Die Medizin liebe die Heilbaren, nicht die Unheilbaren.

Wenn es ums Wegsperren geht, wird Dörner noch schärfer - gegen sich selbst: In „Bürger und Irre“ habe er den Gedanken schon einmal gedacht, dass die Psychiatrie „soziale Euthanasie“ betrieben habe und die Nazis die tatsächliche Euthanasie nur „draufgesattelt“ hätten. Doch das Verrückte: „Als ich mich selbst hinein begeben habe in die Praxis der Psychiatrie, ist es mir wahrhaftig gelungen, diesen Gedanken jahrzehntelang zu verdrängen. Erst als der Aachener Professor Frank Schneider 2010 als Präsident des Psychiatrischen Dachverbands DGPPN in einer historischen Rede die Frage aufwarf, ob die Euthanasie mehr ein Naziprogramm oder mehr ein Psychiatrieprogramm gewesen sei, habe er sich wieder an seine damaligen Gedanken erinnert.
„Ende der Veranstaltung“, das ihm wichtigste Buch, wurde weitgehend ignoriert, aber Dörner hat nicht resigniert. Im Gegenteil. Das hat ihn angestachelt. So hat sich der Mann wieder an seine Rheinmetall-Schreibmaschine gesetzt, an der schon die beiden Doktorarbeiten entstanden sind, und hat für die Neuauflage im Sommer 2015 ein neues Vorwort geschrieben.

\section{Bei der Inklusion darf Zeit keine Rolle spielen.}

Selbstkritik auch darin: Der Erfolg der Integration wäre noch größer gewesen, hätte man sich damals noch zwei, drei Jahre mehr Zeit gelassen. Seine Lehre für heute: „Bei der Inklusion darf Zeit keine Rolle spielen." Und sie müsse auch für Flüchtlinge gelten, die hätten auch ein Recht, in normalen Wohnungen zu leben. Solche Sätze hackt Dörner nicht in den Computer. Er hat keinen. Und er will keinen. Wer mit ihm korrespondieren will, erhält getippte Postkarten und kann ihn anrufen. Er steht im Telefonbuch.

Dass der Mann beharrlich am Bewährten festhält, dass es mindestens einen Verlag gibt, der seine radikalen Forderungen druckt, hat auch mit jenem Bild zu tun, das am schlichten Fichtenholzschrank gleich neben dem Eingang zum Arbeitszimmer klebt. Kaum hat man sich erkundigt, wer die altmodisch gekleideten Herren darauf seien, steht man schon davor, und Dörner deutet auf den melancholisch dreinblickenden Herrn links. Jakob van Hoddis. Dichter des Expressionismus, „Das Weltende“. „Dem Bürger fliegt vom spitzen
Kopf der Hut“, hebt Dörner an, „in allen Lüften hallt es wie Geschrei ...“.

Dieser van Hoddis, erklärt der Gastgeber, hatte einen dreifachen Makel: „Er war Jude, entarteter Dichter, und psychisch krank.“ Nach langen Jahren in Nervenheilanstalten wurde van Hoddis 1942 vermutlich in Sobibor ermordet. Er ist der Namenspatron des Verlags, den Dörner mit seinem Gütersloher Team 1986 gründete. Darin erschienen Dörner-Titel wie „Ende der Veranstaltung“, „Tödliches Mitleid“ oder „Überlegungen im Warteraum zum Gas“.

Die Konfrontation mit dem Holocaust ist ein starker Motor in Dörners Leben. Der drehte erstmals heiß, als der junge, damals noch marxistisch orientierte Assistenzarzt in Eppendorf auf Hans Bürger-Prinz traf. Der damalige Eppendorfer Klinikleiter war zwar konservativ gewesen, verschaffte dem hochbegabten Nachwuchsmediziner und Soziologen aber die Gelegenheit, Gutachten zu schreiben. Und der nahm das Entgegenkommen dieses „Militärarschs - des heißgeliebten“ gerne an. So konnte Dörner hunderte von Patienten-Dokumentationen aus der Nazizeit auswerten. Bis heute ist er getrieben davon, die Verbrechen der Nazis sichtbar zu machen.

\section{Ein Kampf gegen alle.}

Als Dörner nach elf Jahren an der Uniklinik Hamburg nach Gütersloh ging, waren dort nicht alle begeistert. Professor Walter Theodor Winkler, früherer Leiter der Klinik, Vorsitzender der Allgemeinen Ärztlichen Gesellschaft für Psychotherapie (AÄGP) und Mitglied der Enquête-Kommission, ließ kei- 
men musste. Noch heute ist Dörner überzeugt: „Es hat die permanente Anwesenheit bei Tag und bei Nacht gebraucht.“ Denn es war „ein Kampf gegen alle, ein Krieg“. Die betroffenen Patienten, die Angehörigen, die Betriebsleitung, der kaufmännische Leiter, der Pflegedienstleiter, der Personalrat, der Träger - alle hatten sie mehr oder weniger Angst um ihre Existenz.

Unbequem wurde es zunächst auch für seine Frau, eine Krankenschwester, die er in der Eppendorfer Uniklinik kennengelernt hatte. Die heute 71-Jährige wollte nicht weg von Hamburg in die westfälische Provinz. Doch der Charismatiker überzeugte sie. Man vereinbarte: Wann immer sie es nicht mehr aushalten würde, würde er unmittelbar kündigen und mit ihr zurückgehen. Dann überzeugte er alle anderen - Kostenträger, Kollegen, Arbeitgeber, Patienten, Angehörige, Nachbarn.

Paul Dörner, 41, examinierter Krankenpfleger und tätig an der einstigen Wirkungsstätte seines Vaters in Gütersloh, kann sich noch gut erinnern an die Zeit, als er an Heiligabend mit dem Papa durch die gesamten Stationen spazierte. Der stattete jedem Patienten einen Besuch ab. Manche von ihnen hatten ihn schon für verrückt erklärt, als er sich bei seinem Einstand als Klinikleiter bei jedem einzeln vorstellte.

Und Ende der 1980er zogen die Dörners sogar im selben Viertel um, auf dass Platz für eine ambulante Betreuung entstehe. Paul Dörner nennt es die Zeit des „Enthospitalisierungseifers“. Das elterliche Umfeld scheint den Kindern aber nachhaltig gefallen zu haben: Dörners Tochter Kathrin arbeitet in der ambulanten Nachbarschaftsinitiative „Alt und Jung Süd-West e. V.“ Bielefeld, ebenfalls als Krankenschwester; Sohn Henry ist Arzt.

\section{Die Entdeckung des Trialogs war ein Resultat der Not.}

Klaus Dörner lehnt sich entspannt zurück, ermuntert den Gast, sich bei der bewährten zuckerfreien Limonade zu bedienen, die er und seine Frau auf ihren mehrmonatigen Australienreisen schätzen gelernt haben. Gleich nach der Pensionierung wa- ren sie dort zum ersten Mal, Dörner wollte für niemanden mehr greifbar sein und überließ es seiner Frau, ihn auf eine Reise mitzunehmen, deren Ziel er erst am Flughafen erfuhr. Hauptsache so weit wie möglich weg, das war seine Vorgabe. Die Nachfolger sollten ihren eigenen Weg gehen.

Und wieder blendet Dörner das Licht auf sein Denkmal ein wenig ab. Etwa, wenn die Sprache auf den „Trialog“ kommt, die „Entdeckung der Angehörigen“, wie Dörner es milde lächelnd nennt. Die war ein Resultat der Not: Gemeinsam mit seiner Co-Autorin Ursula Plog baute er am Uniklinikum Eppendorf (UKE) eine Tagesklinik auf, doch Schizophrene, Manisch-Depressive oder chronisch Suchtkranke konnte man nachmittags nicht einfach so - statt auf die Station - nach Hause schicken. Man musste herausfinden, wie die Angehörigen tickten. Wenn es heute heiße: Dörner hat die Angehörigengruppen entdeckt, „dann schämt man sich heute fast dafür. Es war ja nur zur eigenen Absicherung“.

Der Impuls war dennoch der richtige. Denn was sie da zu hören bekamen, weitete die Sinne für die wahre Problematik: „Die Angehörigen haben damals zum ersten Mal ihr Maul aufgemacht - und wurden gehört. Wir hatten gar keine Ahnung, wie demütigend wir mit denen umgegangen waren, wenn wir zum Beispiel von der ,schizophrenogenen Mutter" sprachen.“

Oder die Nachbarschaftshilfe, der „Bürger-Profi-Mix“. Eine Zufallsentdeckung: In Hamburg-Altona, zurückgekehrt aus dem politischen Hexenkessel Berlin der 1968er, geschieden von der ersten Frau, einer Historikerin, und getrennt von den beiden Töchtern, bekam der Assistenzarzt Dörner die Gelegenheit, am psychiatrischen Dienst des Gesundheitsamts Altona zu arbeiten. Kein Mediziner hätte sich damals freiwillig auf so eine Behörde eingelassen. Der unkonventionelle Dörner schon, er hatte aber schlicht keine Ahnung, was es hieß, einmal pro Woche halbtags allein verantwortlich zu sein für zig chronisch psychisch Kranke.

Doch da waren diese beiden bodenständigen Fürsorgerinnen, wie sie früher hießen. „Die beiden waren großartig.“ Sie öffneten ihm die Augen, wie man chronisch Kranke betreut, die in der eigenen Wohnung leben. „Ich verstand, dass das nur geht, wenn man normale Bürger in Nachbarn verwandelt.“

\section{Jeder braucht eine Tagesdosis Bedeutung für einen anderen.}

Zum Beispiel so: Die alleinerziehende Mutter eines Zwölfjährigen muss wegen eines depressiven Schubs schon wieder nach Ochsenzoll. Wohin mit dem Sohn? Spätestens beim zehnten Nachbarn hat es funktioniert und der Junge wurde betreut. „Damals habe ich erstmals von der Existenz der Rasse der Nachbarn erfahren“, scherzt Dörner, „ich hatte gedacht, die wären im Mittelalter ausgestorben“. Mehr denn je ist er inzwischen davon überzeugt, dass jeder Mensch „eine Tagesdosis Bedeutung für einen anderen braucht“, die Menschen wollen sich in den Dienst anderer stellen - mit der nötigen professionellen Unterstützung.

Beim Verabschieden kommt der schwarz-weiße Kater aus der Wohnküche und gibt noch einmal Anlass zu einem letzten Akt Dörner'scher Selbstkundgabe. Zorro heißt er, wie der Rächer der Armen im Abenteuerroman. Als Zorro den Gästen um die Beine streicht und schnurrt, fragen wir, ob es nicht artgerecht wäre, der liebesbedürftigen Seele eine Partnerin zu verschaffen. Da lacht Dörner. Sie hatten früher zwei Hunde, zwei Katzen und einen Papagei. Jetzt nochmal ein junges Tier? Das wäre doch unfair dem Tier gegenüber, wenn es dann allein wäre. - „Dann“? Kann man beiläufiger über das eigene Ende reden?

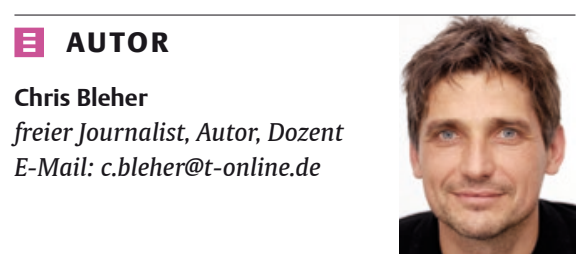

\section{BIBLIOGRAFIE}

DOI 10.1055/s-0041-108128

Psych Pflege 2016; 22: 7-11

(c) Georg Thieme Verlag KG

Stuttgart · New York · ISSN 0949-1619 